\title{
Sonographic assessment of abdominal fat distribution during the first year of infancy
}

Christina Brei', Daniela Much', Ellen Heimberg², Verena Schulte' ${ }^{1}$, Stefanie Brunner', Lynne Stecher', Christiane Vollhardt', Jan S. Bauer ${ }^{3}$, Ulrike Amann-Gassner ${ }^{1}$ and Hans Hauner ${ }^{1,4}$

BACKGROUND: Longitudinal data regarding the fat distribution in the early postnatal period is sparse.

METHODS: We performed ultrasonography (US) as a noninvasive approach to investigate the development of abdominal subcutaneous (SC) and preperitoneal (PP) fat depots in infants $\leq 1 \mathrm{y}$ and compared longitudinal US data with skinfold thickness (SFT) measurements and anthropometry in 162 healthy children at $6 \mathrm{wk}, 4 \mathrm{mo}$, and 1 y postpartum.

RESULTS: US was found to be a reproducible method for the quantification of abdominal SC and PP adipose tissue (AT) in this age group. Thickness of SC fat layers significantly increased from 6 wk to 4 mo and decreased at 1 y postpartum, whereas PP fat layers continuously increased. Girls had a significantly higher SC fat mass compared to boys, while there was no sexspecific difference in PP fat thickness. SC fat layer was strongly correlated with SFT measurements, while PP fat tissue was only weakly correlated with anthropometric measures.

CONCLUSION: US is a feasible and reproducible method for the quantification of abdominal fat mass in infants $\leq 1$ y of age. PP and SC fat depots develop differentially during the first year of life.

C hildhood obesity has become a global epidemic (1) and there is growing evidence that the first year of life, a phase of rapid growth, constitutes a critical period for the onset of obesity in later life (2). Many studies among adults suggest that potential health risks of obesity such as cardiovascular disease or type 2 diabetes do not only depend on the amount of body fat, but also on the type of fat distribution. Abdominal fat, especially, has been identified to play a central role for the development of complications because of its close relationship to insulin resistance and metabolic cardiovascular risk factors $(3,4)$. Also in children and adolescents, disturbances of insulin and glucose metabolism as well as signs of an unfavorable lipid profile have been described in relation to abdominal fat (5-7). Thus, the role of abdominal fat distribution during infancy and childhood is gaining recognition.
General growth parameters, such as BMI, skinfold thickness (SFT) measurements, and waist circumference or waistto-hip ratio are widely used as measures of body fatness in infants and children, but they do not directly quantify fat compartments. Measurement of waist circumference offers an indirect and crude alternative for the assessment of body fat distribution, but cannot distinguish between subcutaneous (SC) and visceral fat, respectively. However, this parameter is not as accurate as direct measures like ultrasonography (US), computer tomography (CT), or magnetic resonance imaging (MRI) (8-10). Techniques like MRI or CT are expensive and represent a burden for the child, because these examinations are uncomfortable and time-consuming or expose the infants to radiation (11).

US is an easily accessible, inexpensive, radiation-free, and noninvasive approach to measure abdominal adipose tissue (AT). Holzhauer et al. (12) proposed ultrasound as an adapted technique to measure SC and preperitoneal (PP) fat depots in a cross-sectional study of $2121-y$ and 227 2 -y olds, respectively, a method which was described by Suzuki et al. (13) in 1993. It could be shown that US provides a reliable and reproducible estimate of SC and PP fat depots when compared to CT in which PP fat mass was found to be related to abdominal visceral fat mass. Therefore, US is a suitable method for epidemiological and clinical approaches $(13,14)$.

To date, the technique of Holzhauer et al. has not been applied to generate longitudinal sonographic data on AT growth in a younger pediatric population $<1 \mathrm{y}$ of age. However, a different protocol for estimating visceral and SC fat in the first year of infancy has been applied by a different study (15). Aim of the present study was to generate longitudinal sonographic data on AT growth during early infancy. US measurements were performed in parallel with SFT measurements and anthropometry to assess how these measures are correlated. For this purpose, the US technique described originally by Holzhauer et al. (12) was adapted slightly for use in very young infants ( $\leq 1 \mathrm{y}$ of age). 


\section{RESULTS}

\section{Participant Characteristics}

The analyses included 162 infants ( $n=77$ girls, $n=85$ boys) aged $6 \mathrm{wk}$ (median $6.33 \mathrm{wk}$ ) and 160 infants ( $n=79$ girls, $n=81$ boys) aged $4 \mathrm{mo}$ (median $3.64 \mathrm{mo}$ ) who underwent sonographic examinations as well as anthropometric and SFT measurements during regular study visits. Overall 160 children $(n=84$ girls, $n=76$ boys) completed the investigations at $1 \mathrm{y}$ of age (median $1.04 \mathrm{y}$ ). The anthropometric and ultrasound data are presented in Tables 1 and 2. All infants were born full-term between the

Table 1. Anthropometric variables and skinfold thickness measurements at age $6 \mathrm{wk}, 4 \mathrm{mo}$, and $1 \mathrm{y}$

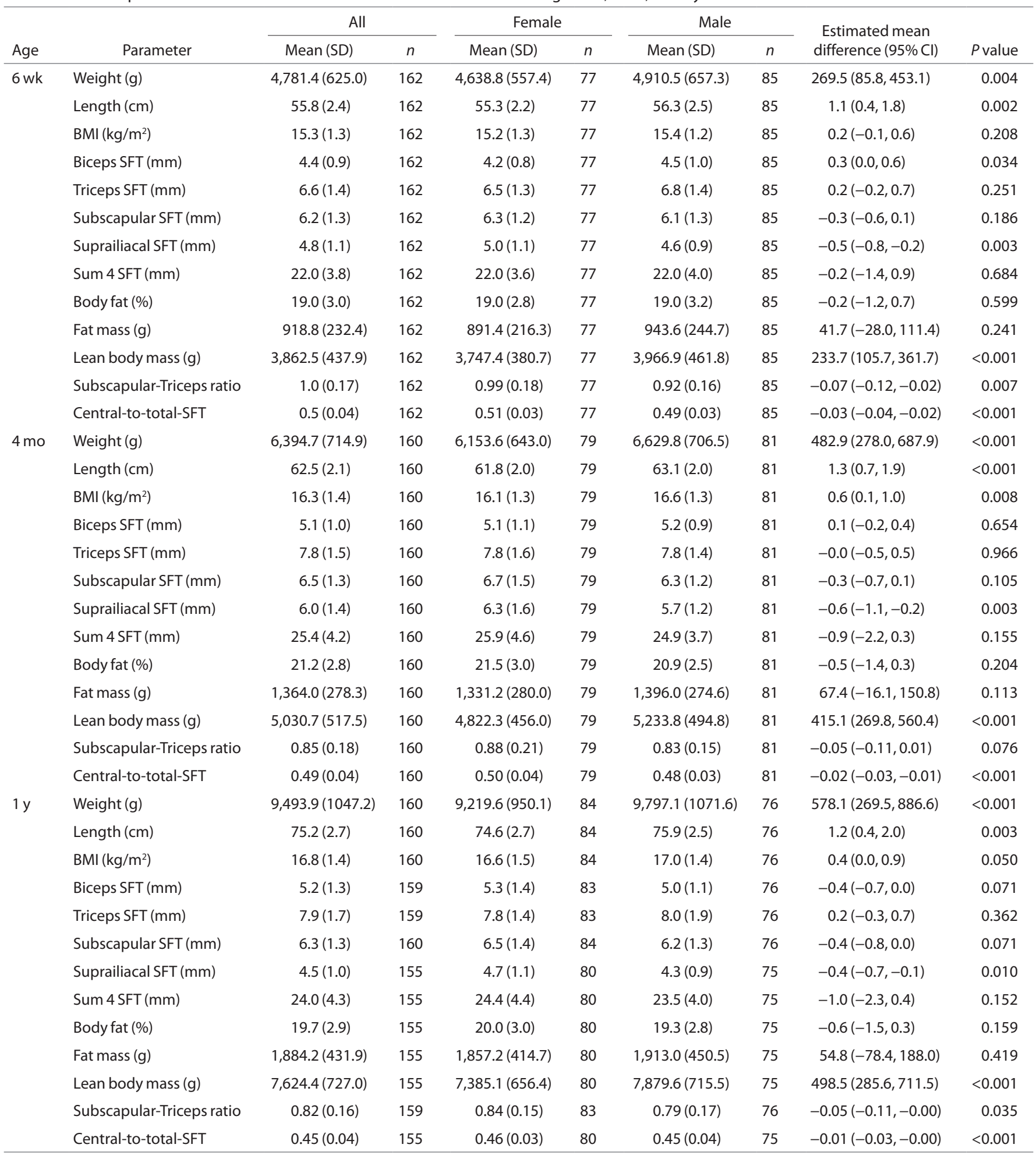

Data are presented as mean \pm SD $(n)$ along with the nonadjusted mean difference ( $95 \%$ confidence interval) from mixed models containing time, sex and an interaction between sex 
37 th and 42 nd week of gestation, except $n=4$ preterm infants and one post-term baby. Sixteen infants were born to mothers suffering from gestational diabetes during pregnancy, which was controlled by diet in 12 cases and treated with insulin in 4 cases.

\section{Reproducibility}

The intra- and interclass-correlation coefficients (ICC), in Table 3, for the observers' estimated distances (mean out of three measurements) and the calculated areas for all measurements suggest strong inter-observer agreement. Additionally, the Bland-Altman plots did not show any relevant differences between observer 1 and 2. On average, the measurements of observer 1 were slightly higher than for observer 2 with a mean difference of $0.38 \mathrm{~mm}^{2}$ for the area of SC fat in sagittal plane (area sag sc) (Figure 1a), $0.81 \mathrm{~mm}^{2}$ for the area of SC fat in axial plane (area ax sc) (Figure 1b) and $0.20 \mathrm{~mm}^{2}$ for the area of PP fat in sagittal plane (area sag pp) (Figure 1c), respectively.
Intra-observer agreement showed comparable results for all measures of the distances and areas of PP and SC fat with ICC ranging from 0.87 (sag caudal pp) to 0.99 (ax r).

When stratified by age group, there was no evidence of a trend in increasing ICC with age (data not shown).

\section{Effect of Respiration}

Breathing phases affect the thicknesses of the fat layers with the greatest thickness at the end of the expiration phase: During inspiration, the liver shifts toward distal direction, reducing the PP fat layer. With increasing expiration, the liver is shifted below the sternum and the layer becomes thicker (data not shown). To consider this effect, measurements were made at the end of expiration using the cine-loop-function.

\section{Effect of Age}

The ultrasound investigations showed pronounced differences in the physiological growth of SC and PP fat depots over the

Table 2. SC and PP fat measurements assessed by US at age $6 \mathrm{wk}, 4 \mathrm{mo}$, and $1 \mathrm{y}$

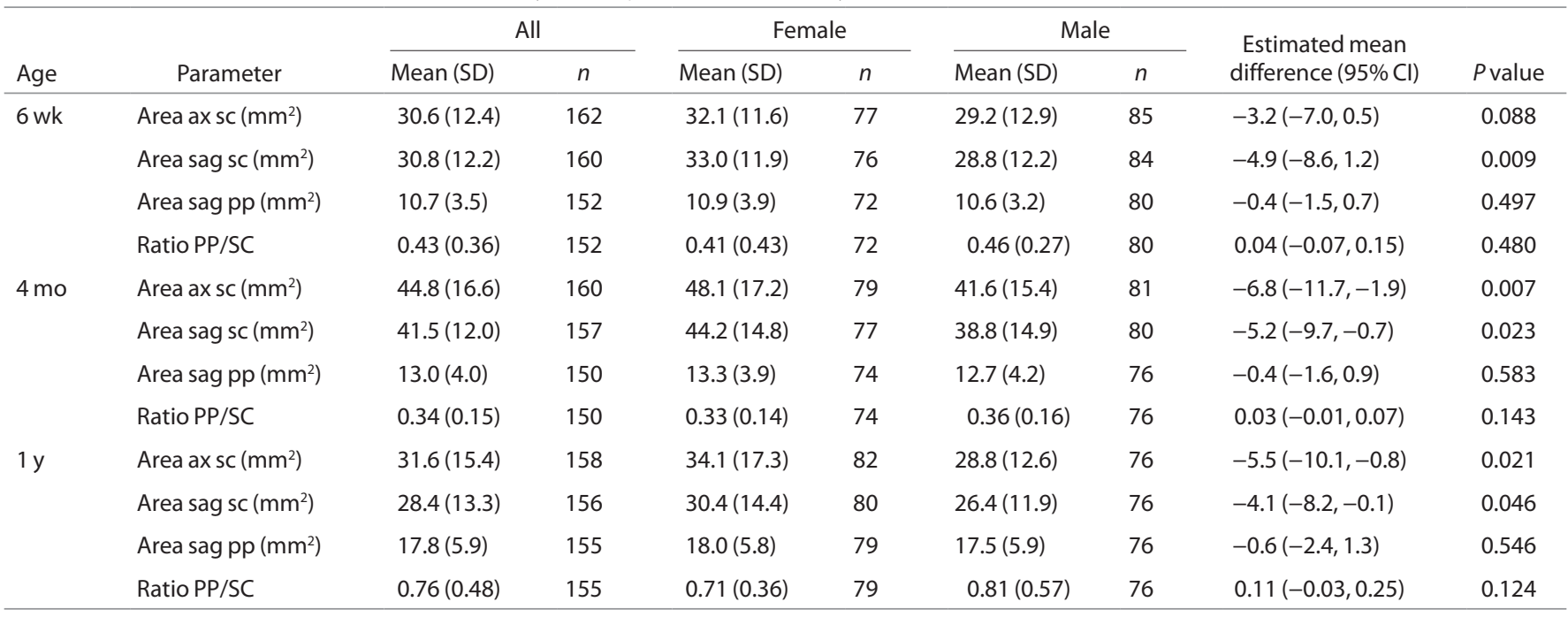

Data are presented as mean \pm SD $(n)$ along with the nonadjusted mean difference ( $95 \%$ confidence interval) from mixed models containing time, sex, and an interaction between sex and time.

PP, preperitoneal; SC, subcutaneous; US, ultrasonography.

Table 3. Inter- and intraclass-correlation-coefficients (ICC) of the precision measurements

\begin{tabular}{|c|c|c|c|}
\hline & & $\begin{array}{c}\text { Inter-observer } \\
\text { agreement ICC (CI 95\%)a }\end{array}$ & $\begin{array}{c}\text { Intra-observer } \\
\text { agreement ICC (Cl 95\%) }\end{array}$ \\
\hline \multirow[t]{7}{*}{ Mean out of three measurements } & sag cranial pp & $0.95(0.91,0.97)$ & $0.98(0.94,1.00)$ \\
\hline & sag caudal pp & $0.94(0.89,0.97)$ & $0.87(0.63,0.96)$ \\
\hline & sag cranial sc & $0.98(0.97,0.99)$ & $0.98(0.93,0.99)$ \\
\hline & sag caudal sc & $0.98(0.97,0.99)$ & $0.98(0.95,1.00)$ \\
\hline & $a x r$ & $0.99(0.97,0.99)$ & $0.99(0.98,1.00)$ \\
\hline & axm & $0.98(0.97,0.99)$ & $0.97(0.92,0.99)$ \\
\hline & $\mathrm{axl}$ & $0.99(0.97,0.99)$ & $0.99(0.97,1.00)$ \\
\hline \multirow[t]{3}{*}{ Area } & Area sag pp & $0.97(0.94,0.98)$ & $0.97(0.90,0.99)$ \\
\hline & Area sag sc & $0.99(0.97,0.99)$ & $0.98(0.95,1.00)$ \\
\hline & Area ax sc & $0.99(0.98,0.99)$ & $0.99(0.97,1.00)$ \\
\hline
\end{tabular}

Data are presented as correlation coefficients (ICC) and corresponding 95\% confidence intervals (CI 95\%); alnter-observer agreement $n=45$; blntra-observer agreement $n=12$. 

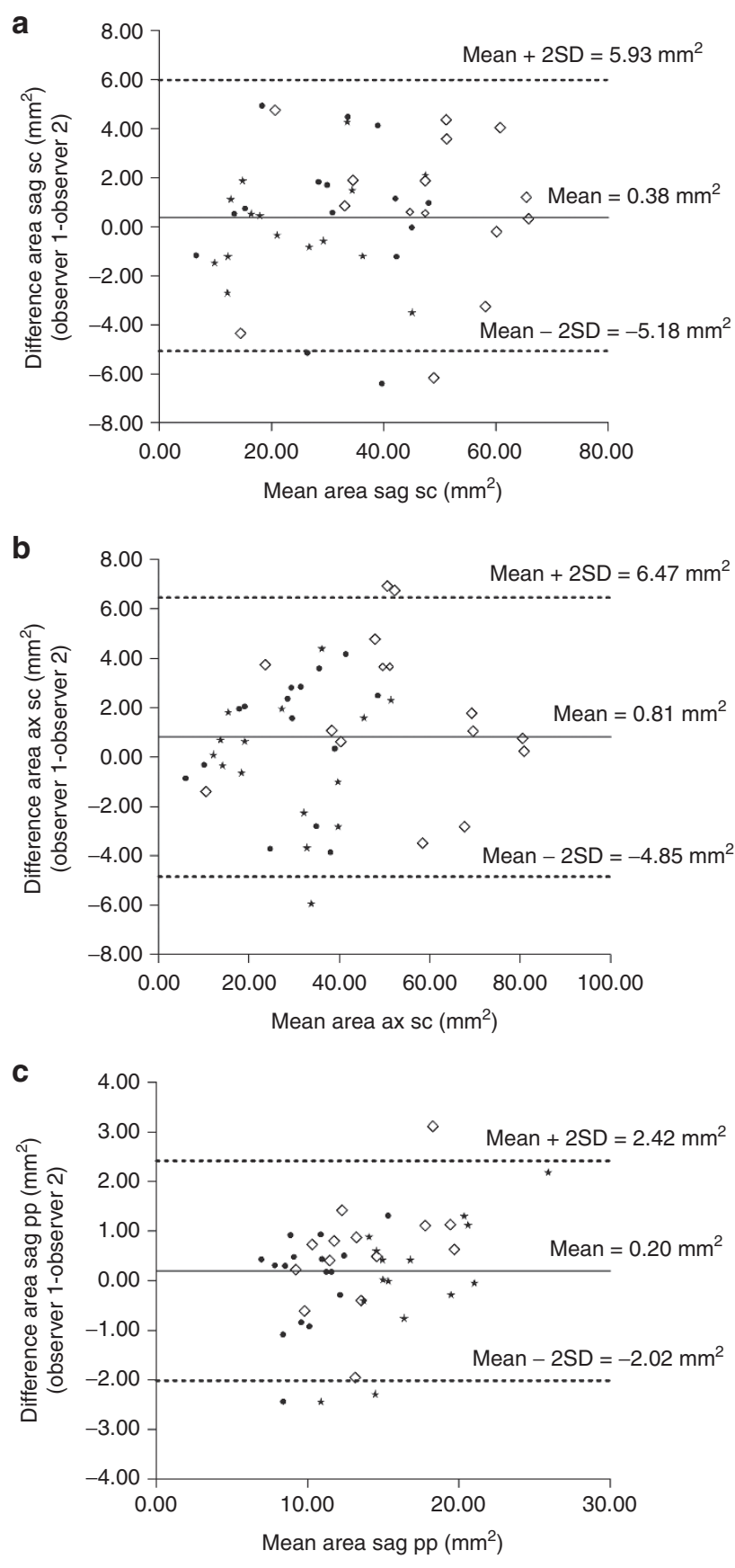

Figure 1. Bland-Altman plots of the area sag sc (a), area ax sc (b), and area sag pp (c), at 6 wk (filled circle), 4 mo (open diamond), and 1 y postpartum (asterisk) of the two observers (observer 1 =E.H., observer 2 = D.M.). The differences between the two observers were plotted against their averages. Average difference and the average difference \pm 2 SD, termed as limits of agreements, are plotted.

first year of life (Table 2 and Figure 2a): Areas of SC fat layers significantly increased from $6 \mathrm{wk}$ (area ax sc $=30.6 \mathrm{~mm}^{2}$, area sag sc $\left.=30.8 \mathrm{~mm}^{2}\right)$ to 4 mo postpartum (area ax sc $=44.8 \mathrm{~mm}^{2}$, area sag $\left.\mathrm{sc}=41.5 \mathrm{~mm}^{2}, P<0.001\right)$ and then significantly decreased toward the first year of life (area ax sc $=31.6 \mathrm{~mm}^{2}$, area sag sc $=28.4 \mathrm{~mm}^{2}, P<0.001$ ).

In contrast, the PP fat layer significantly increased over all measured time points with an estimated mean area sag $\mathrm{pp}$ of
$10.7 \mathrm{~mm}^{2}$ in the 6 -wk olds, $13.0 \mathrm{~mm}^{2}$ in the $4-\mathrm{mo}$ and $17.8 \mathrm{~mm}^{2}$ in the 1-y olds (all $P<0.001$ ). Although, some sex differences in the ultrasound measures were estimated, as discussed in the following section, the same general trend with age was observed (Figure $2 \mathbf{b}-\mathbf{d}$ ).

The ratio PP/SC of the two AT compartments first decreased slightly from 0.43 to 0.34 due to the greater increase in SC fat. Then, the ratio increased sharply to 0.76 reflecting a shift in the abdominal fat tissue-ratio.

There was large variation in the fat layers between the infants for all measuring positions at each time point of investigation. For example, areas ranged from 3.41 to $40.10 \mathrm{~mm}^{2}$ in PP fat and ranged from 4.63 to $71.80 \mathrm{~mm}^{2}$ in SC fat in sagittal plane at $1 \mathrm{y}$ of age.

\section{Effect of Sex}

As shown in Table 2 and Figure $2 b, c$, there is some evidence that females have greater SC fat layers compared to males, particularly at $4 \mathrm{mo}$ and $1 \mathrm{y}$. There is no evidence that $\mathrm{PP}$ fat thickness differs between the sexes (Table 2 and Figure 2d). Consistent with the results measured with US, girls were estimated to have significantly thicker SC fat mass, assessed by higher suprailiac SFT at $6 \mathrm{wk}, 4 \mathrm{mo}$, and 1 y postpartum without consistently significant sex-specific differences in subscapular, biceps, and triceps SFT measurements. The resulting calculated percentage of fat mass was slightly higher at 4 and 12 mo postpartum in girls, but these differences were not statistically significant. Fat distribution was shifted toward a more centralized pattern in the girls compared to the boys: The subscapular-to-triceps SFT ratio was significantly higher in the girls at $6 \mathrm{wk}$ and 12 mo postpartum. In addition, a higher central-to-total SFT ratio was estimated in girls at $6 \mathrm{wk}, 4 \mathrm{mo}$, and 12 mo postpartum (Table 1). However, boys had on average a significantly higher body weight and length at each time point of investigation, attributable to a significantly higher lean mass (all $P<0.001)$.

\section{Correlation Coefficients of the Different Fat Measures Among Each Other and With Anthropometric Measures}

There was strong correlation between the two measures of SC fat at each time point $(r>0.9)$ (Figure 3a). However, the areas calculated in the axial plane were significantly higher at $4 \mathrm{mo}$ and 1 y postpartum (both $P<0.001$ ).

The correlations between the PP AT area and the SC AT areas in sagittal and axial plane were weak (Spearman's rho area sag pp/area sag sc $r=0.24$, area sag pp/area ax sc $r=0.25)$ at $6 \mathrm{wk}$, but increased with increasing age (Spearman's rho at 1 y of age: area sag pp/area sag sc $r=0.47$, area sag pp/area ax sc $r$ $=0.47$ ). A scatterplot showing the association between SC and $\mathrm{PP}$ in sagittal plane is presented in Figure $3 \mathbf{b}$.

PP fat showed weak correlations with the anthropometric measurements at the time points $6 \mathrm{wk}$ and $4 \mathrm{mo}$. The correlation increased slightly at $1 \mathrm{y}$ for PP fat tissue and subscapular $(r=0.41, P<0.001)$, suprailiacal $(r=0.31, P<0.001)$, and sum 4 SFT $(r=0.38, P<0.001)$. The association between PP fat layers and sum 4 SFT and BMI is shown in Figure 3c,d, respectively. 


\section{Articles | Brei et al.}
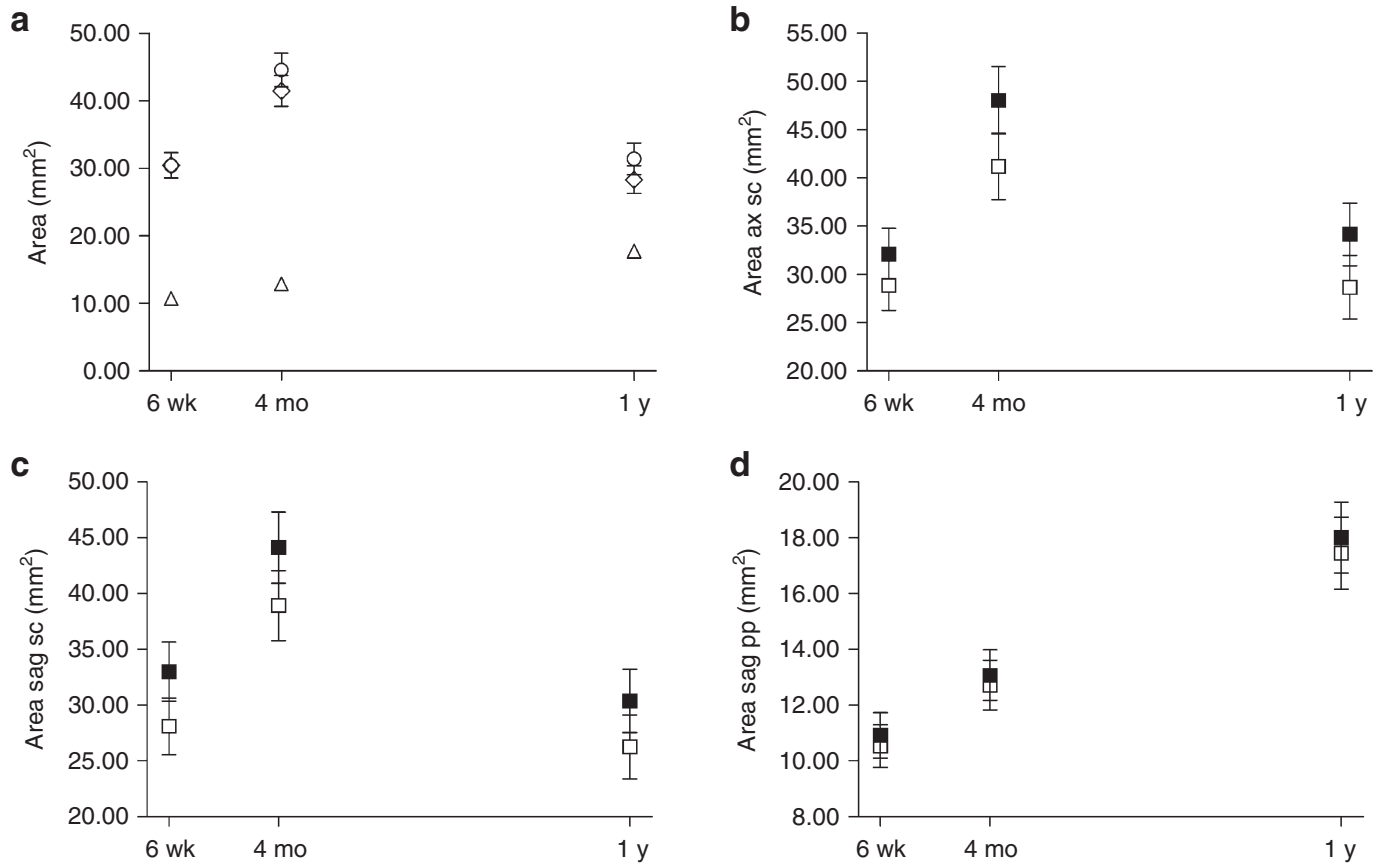

Figure 2. Effect of age and sex on subcutaneous and preperitoneal fat tissue compartments, stratified by the time point of investigation. (a) Comparison of area ax sc (circle), area sag sc (diamond), and area sag pp (triangle) in $6 \mathrm{wk}, 4 \mathrm{mo}$, and 1 -y-old infants. Estimated means and $95 \%$ confidence intervals are from a mixed linear model with time as a fixed effect. (b-d) Effect of sex on area ax sc (b), area sag sc (c), and area sag pp (d) in 6 wk, 4 mo, and 1-y-old females (filled squares) and males (open squares). Estimated means and 95\% confidence intervals are from a mixed linear model with time, sex and an interaction between sex and time as fixed effects.
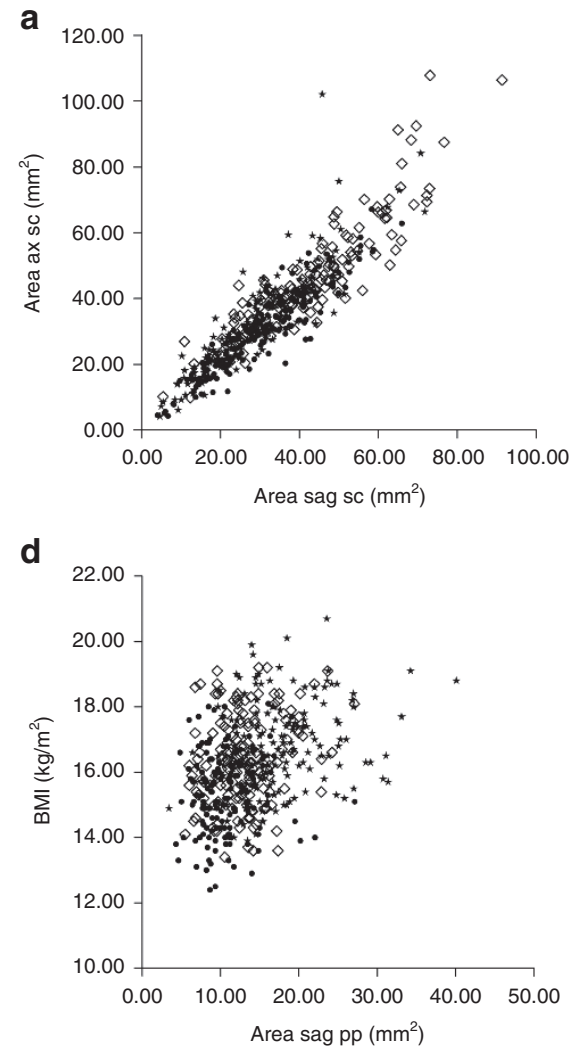
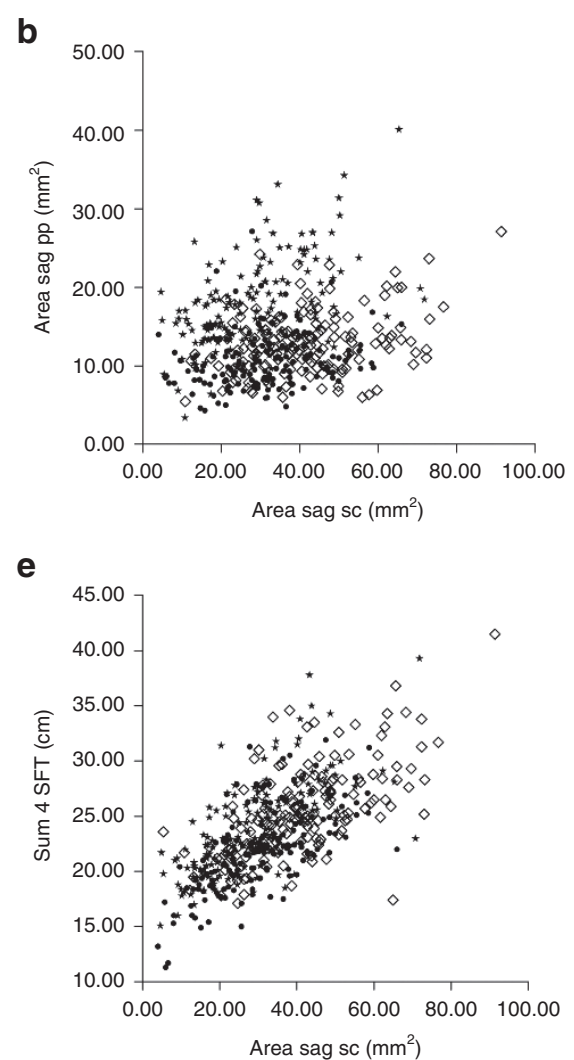

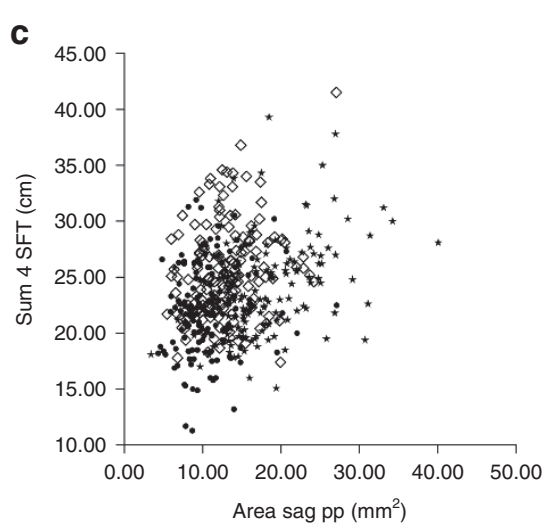

f

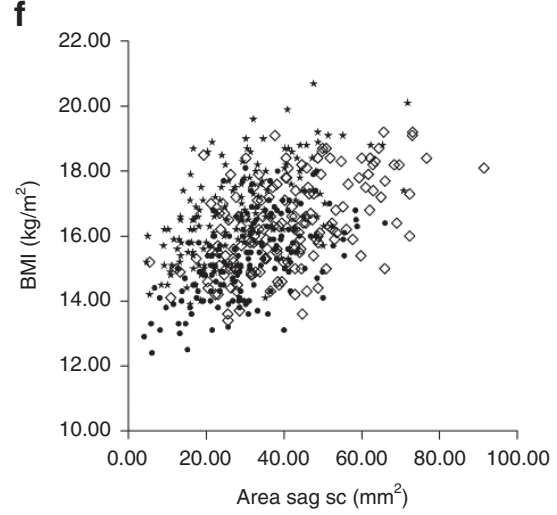

Figure 3. Scatterplots showing the association between subcutaneous (SC) adipose tissue (AT) in sagittal and axial plane (a), preperitoneal and SC AT in sagittal plane (b) and between area sag pp (c,d) area sag sc (e,f) and anthropometric measures at 6 wk (filled circle), 4 mo (open diamond), and 1 y postpartum (asterisk). 
In contrast, the sonographic measures of $\mathrm{SC}$ fat tissue were moderately correlated with body weight, length, and BMI at all three time points. They were highly correlated with the sum 4 SFT, especially at 1 y postpartum (area sag sc $r=0.72$, area ax sc $r=0.71$, Table 4), subscapular (area sag sc $r=0.70$, area ax sc $r=0.68$ ), and suprailiac SFT (area sag sc $r=0.69$, area ax sc $r=0.70$ ), whereas biceps and triceps SFT measurements showed weak to moderate correlations during the assessment period. Associations at each time point between SC fat layers and sum 4 SFT and BMI are shown in Figure 3e,f, respectively.

\section{DISCUSSION}

The aim of the present study was to characterize how abdominal PP and SC fat depots, assessed with US, change over the first year of infancy and to compare these measures with anthropometric measurements. Therefore, we adapted slightly the technique of sonographic assessment of PP and SC fat, previously described by Holzhauer et al., (12) for our cohort of infants $\leq 1$ y of age.

Table 4. Spearman-correlation-coefficients between ultrasound and anthropometric measures

\begin{tabular}{|c|c|c|c|c|}
\hline $6 w k$ & Area sag pp & Area sag sc & Area axsc & Ratio PP/SC \\
\hline Weight & $0.17^{*}$ & $0.54^{+}$ & $0.56^{+}$ & $-0.41^{\dagger}$ \\
\hline Length & 0.14 & $0.27^{\dagger}$ & $0.25^{+}$ & $-0.22^{* *}$ \\
\hline BMI & $0.10^{\mathrm{a}}$ & $0.57^{t, a}$ & $0.60^{+}$ & $-0.43^{+a}$, \\
\hline Biceps & $0.19^{*}$ & $0.39^{+}$ & $0.34^{+}$ & $-0.25^{* *}$ \\
\hline Triceps & $0.18^{*}$ & $0.51^{+}$ & $0.50^{+}$ & $-0.33^{+}$ \\
\hline Subscapular & 0.11 & $0.60^{+}$ & $0.64^{+}$ & $-0.48^{+}$ \\
\hline Suprailiacal & $0.21^{* *}$ & $0.65^{+}$ & $0.63^{+}$ & $-0.47^{\dagger}$ \\
\hline Sum 4 SFT & $0.20^{*, a}$ & $0.66^{t, a}$ & $0.65^{+}$ & $-0.46^{t, a}$ \\
\hline $4 \mathrm{mo}$ & Area sag pp & Area sag sc & Area axsc & Ratio PP/SC \\
\hline Weight & 0.14 & $0.40^{+}$ & $0.43^{+}$ & $-0.28 \dagger$ \\
\hline Length & -0.05 & 0.02 & 0.04 & -0.12 \\
\hline BMI & $0.19^{* a}$ & $0.49^{+, \mathrm{a}}$ & $0.52^{+}$ & $-0.28^{t, a}$ \\
\hline Biceps & 0.09 & $0.30^{+}$ & $0.34^{+}$ & $-0.19^{*}$ \\
\hline Triceps & 0.06 & $0.48^{+}$ & $0.48^{+}$ & $-0.36^{\dagger}$ \\
\hline Subscapular & $0.22^{* *}$ & $0.53^{+}$ & $0.53^{+}$ & $-0.29^{\dagger}$ \\
\hline Suprailiacal & $0.25^{* *}$ & $0.66^{+}$ & $0.68^{+}$ & $-0.40^{+}$ \\
\hline Sum 4 SFT & $0.21^{* a}$ & $0.63^{+, a}$ & $0.65^{+}$ & $-0.39^{t, a}$ \\
\hline $1 y$ & Area sag pp & Area sag sc & Area axsc & Ratio PP/SC \\
\hline Weight & $0.25^{* *}$ & $0.46^{+}$ & $0.41^{+}$ & $-0.31^{\dagger}$ \\
\hline Length & 0.10 & 0.02 & -0.04 & 0.06 \\
\hline BMI & $0.26^{\mathrm{a}}$ & $0.58^{\mathrm{a}}$ & 0.56 & $-0.44^{t, a}$ \\
\hline Biceps & $0.22^{* *}$ & $0.48^{+}$ & $0.46^{+}$ & $-0.33^{+}$ \\
\hline Triceps & $0.25^{* *}$ & $0.45^{+}$ & $0.44^{+}$ & $-0.30^{+}$ \\
\hline Subscapular & $0.41^{+}$ & $0.70^{+}$ & $0.68^{+}$ & $-0.45^{+}$ \\
\hline Suprailiacal & $0.31^{+}$ & $0.69^{+}$ & $0.70^{+}$ & $-0.50^{\dagger}$ \\
\hline Sum 4 SFT & $0.38^{t, a}$ & $0.72^{+, a}$ & $0.71^{+}$ & $-0.48^{t, a}$ \\
\hline
\end{tabular}

Regarding AT growth, we observed a significant increase of SC fat layers from $6 \mathrm{wk}$ to $4 \mathrm{mo}$ and a decrease until $1 \mathrm{y}$ postpartum. Our results suggest a different pattern for the development of PP fat, which increased from $6 \mathrm{wk}$ postpartum up to the age of $1 \mathrm{y}($ all $P<0.001)$. There is some evidence that girls have higher SC fat mass compared to boys, but there was no evidence of a sex-specific difference in PP fat thickness. While the SC fat layer assessed by US was strongly correlated with SFT, PP fat tissue was only weakly correlated with conventional anthropometric measures. In addition, we could show for the first time that US is a reproducible method for the quantification of abdominal SC and PP AT in this age group.

A major strength of our study is the longitudinal study design and the relatively large and consistent number of subjects over the survey period. Despite the very young study sample, the results from intra- and inter-observer-analyses and the BlandAltman plots showed very good agreement and were comparable with the findings of the study of Holzhauer et al. (12), although we have followed a slightly different approach. While the reproducibility in Holzhauer's paper refers to taking the ultrasound images, we have examined it by analyzing the ultrasound images. However, both approaches showed very good results, indicating a good reproducibility of the method.

Healthy infants gain body fat during their first months of life and SC AT composes the main part of total body fat in the first year postpartum, varying between 89.0 and $92.8 \%$ (16). The percentage of total body fat reaches a maximum between 3 and 6 mo postpartum and then slowly decreases during the second half of the first year of life $(17,18)$. This is reflected in our data combining direct and indirect methods and might be due to an increased physical activity at about 6 mo when the crawling phase begins. Holzhauer et al. (12) found a pronounced increase of $45 \%$ in the thickness of the PP fat layer during the second year of life, whereas the SC AT showed no increase. Consequently, this resulted in a shift in the abdominal tissue-ratio toward an increase in PP fat mass. We could show that the shift in abdominal fat distribution toward more PP fat already occurs before the age of $1 \mathrm{y}$. Assuming that PP fat is an approximation of intra-abdominal fat in children (14), our results are consistent with another study which reported a $20 \%$ increase in visceral fat between the third and twelfth month postpartum, assessed sonographically (15). Olhager et al. (19) also showed a significant increase in nonsubcutaneous fat layers within the first 4 mo of infancy with MRI scans. Our observations of differential changes in abdominal fat suggest that the two different fat layers develop independently.

Sex differences in the pattern of fat distribution are well known in adults, with women having greater SC and less visceral AT than men $(10,20,21)$. Regarding SC fat, some authors have shown that differences between sexes already occur in childhood from the first year of life on (12,22-25), which could also be demonstrated by our findings. These observations differ from studies that provided no evidence of gender-specific differences under the age of ten $(22,25)$. Regarding PP fat, sex differences in visceral fat seem to be closely linked to age- and puberty-related changes in fat distribution $(8,22)$. However, the 
question remains open when such differences in fat patterning first emerge because data in early childhood show conflicting results $(9,12,15,22,23,25,26)$. Further follow-up investigations in children, especially for internal fat, are needed.

Within the first year of life, we found SC fat thickness by US highly correlated with the sum 4 SFT and abdominal SFT, particularly at $1 \mathrm{y}$ of age. The associations with BMI and weight were less pronounced. In contrast, PP fat was rather found to be weakly correlated with these anthropometric measurements. As SFT refers to the measurement of SC fat, this explains why stronger correlation was observed with SC fat than PP fat. Our results are in line with the observations from Liem et al. (27), who showed in 6- to 7-y-old healthy children that the sum of suprailiac and abdominal skinfolds was most strongly associated with SC abdominal AT, assessed by CT, followed by abdominal skinfolds, BMI, suprailiac skinfold, hip, and waist circumferences.

Also in the study of Holzhauer et al., where skinfolds have not been considered, BMI showed only a moderate association with SC fat layers. However, in a meta-analysis of the pediatric literature with 497 children aged 7-16 y, Brambilla et al. (8) identified waist circumference as the best predictor for intraabdominal fat mass and BMI as the best single predictor of SC fat although skinfolds as a predictor were not considered. However, our SFT measurements, especially the sum of 4 SFT and abdominal SFT measurements show stronger correlations with SC fat areas directly measured by US than BMI.

There are some limitations of our study. Although the adaptation to the technique of Holzhauer et al. (15) was minor, our study is lacking a gold standard for comparison such as MRI in this specific age group. To definitively establish this US technique for the assessment of fat distribution in early infancy, an age-specific validation with other direct methods is needed. Furthermore, the study population consisted mostly of German children with a BMI in the normal range. Therefore, the results cannot be generalized to other ethnic groups or to over- or underweight children. A clear differentiation of AT layers with the stated anatomical reference structures is not always possible; there remained some technical difficulties, mostly due to the young age of the participants. For example, the restlessness of the infants made the procedure challenging. Another problem was caused by the high breathing-intensity of the infants, with frequencies of $25-30 / \mathrm{min}$, compared to older children or adults and the influence of liver movements on PP fat thickness. However, it was still possible to obtain high-quality images, which was also reflected by high intraand interclass-correlation coefficients.

Although direct methods, such as CT and MRI represent the gold standard for the assessment of SC and PP AT, they have only a limited application for scientific research in infants. Reasons for that (i.e., apart from the cost- and time-consuming certainty), include a high sensitivity to breathing motions (i.e., breath-holding techniques are not feasible in this age group), the need for expensive measurement equipment, handled by suitably trained personnel and the exposure to radiation (CT only) (12,15). Mook-Kanamori and colleagues compared in a group of 34 nonobese children with a median age of $9.5 \mathrm{y}$ (95\% range 0.3-17.0 y) SC and PP fat thickness and areas by CT and US. Correlation coefficients ranged from $0.75-0.97$ (all $P<0.001)$. Two other studies used a different sonographic approach to assess SC and intra-abdominal AT and performed validation studies with children $(n=31$, range 6.0-7.9 y) (27) and newborns ( $n=22$; range 6-19 d) (15). They validated US against MRI measurements and showed moderate to strong positive correlations. However, to definitively establish US for the assessment of fat distribution in children, an additional age-specific validation with a larger sample size for each age group is required.

In summary, our data suggest that US is a feasible method with good reproducibility for the quantification of abdominal SC and PP AT in early infancy. Especially, the latter was described as a discretely developing fat depot. Our results clearly indicate a differential growth of both fat depots towards an increase in PP fat mass during the first year of life. Further studies of longitudinal design, with different assessments over the first year of life and beyond are warranted, to characterize the temporal pattern of AT development at the specific anatomical locations. By associating this data with metabolic parameters, this information may allow a better prediction and prevention of disease risk early in life.

\section{METHODS}

\section{Study Population}

This analysis was embedded in the INFAT-study, a randomized, controlled trial primarily designed to investigate the effect of fatty acids in maternal nutrition during pregnancy and lactation on infant AT development within the first year of life. Rationale, study design, and the clinical results up to $1 \mathrm{y}$ of age have been described in detail elsewhere $(28,29)$. The study population consisted of 208 healthy pregnant women of Caucasian origin (99.5\%) and their newborns, living in the area of Munich, Bavaria, Germany and recruited between July 2006 and May 2009. As there were no significant differences between the study groups with respect to infant body composition (28), study groups were pooled for the following analysis. For the present analysis, only 162 infants with available ultrasound data at $6 \mathrm{wk}$ postpartum were included. The ethical committee of the Technische Universität München (No. 1479/06/2006/2/21) approved the study protocol. Written informed consent was obtained from all participating mothers.

\section{Data Collection and Anthropometric Measurements}

Anthropometric data and SFT were obtained by trained research assistants at $6 \mathrm{wk}, 4 \mathrm{mo}$, and 1 y postpartum as previously described (28). In brief, the infants' weight and length were measured and BMI $\left(\mathrm{kg} / \mathrm{m}^{2}\right)$ was calculated. SFT measurements were performed in triplicate under standard conditions with a Holtain caliper (Holtain, Croswell, Crymych, UK) at the left body axis at four sites (triceps, biceps, subscapular, suprailiac). The mean of the triplicate measurements was used for analysis. The calculation of body fat (\%) was done via predictive skinfold equations according to the method of Weststrate et al. (30). Additionally, we calculated the sum of the four skinfolds and two indices of fat patterning: the subscapular-to-triceps skinfold ratio as an index of central to peripheral fat distribution (31) and the central-to-total skinfolds ratio (trunk-to-total skinfolds \%) using the equation (subscapular + suprailiac)/(sum 4 SFT)^100 (32).

\section{Sonographic Assessment of Abdominal Subcutaneous and Preperitoneal Fat}

The ultrasound investigations were performed using a highresolution ultrasonographic system (Siemens Acuson Premium, Munich, Germany). Measurements were performed by two trained 
research pediatricians (E.H., V.S.). Abdominal SC and PP fat thickness, the latter considered to be an approximation of visceral/intraabdominal fat (12), were measured with a $10 \mathrm{MHz}$ linear probe (VFX 13-5, Siemens Medical Solutions, Erlangen, Germany) in b-picturemode. The infants were located in supine position. Care was taken to minimize movements of the infant. The probe was placed on the skin surface of the upper abdomen of the infant without compression of the tissue layers. We defined two areas of measurement: To determine PP and SC fat, the first measurement was performed in sagittal plane in the middle of the xiphoid process. The second measurement was performed in axial plane, in between the xiphoid process and the umbilicus to determine the SC fat layer.

In previous studies in children or adults $(27,33)$, the measurements were performed at the end of a gentle expiration, however, this procedure is not applicable in a young pediatric population. To get a standardized breathing-phase the cine-loop-function was used. By this function, it is possible to save the last 63 pictures taken and after "defreezing", all individual pictures can be displayed. Thereby, it was possible to identify retrospectively single pictures taken at the end of expiration with tissue layers as much as possible in parallel. The images were stored at an off-line working station for evaluation (Apple Power PC G4, Apple, Cupertino, CA).

\section{Evaluation of the Ultrasound Pictures}

The size of each individual fat layer was determined with the OsiriX software (http://www.osirix-viewer.com, Genf, Schweiz) in both planes. The evaluation process was performed off-line by two examiners (E.H., D.M.) in a blinded fashion after selecting the three most appropriate pictures in sagittal and axial plane for each case.

Preperitoneal fat. Preperitoneal fat was defined as distance between the linea alba as the upper border until the peritoneum located at the upper margin of the liver as the lower border. The first measurement point was set $0.5 \mathrm{~cm}$ caudal from the xiphoid process (sag cranial pp), appearing as a hypoechoic cartilaginous structure, while the second measurement point was set $1.0 \mathrm{~cm}$ caudal from the first reference point (sag caudal pp) (Figure 4).

In each patient, three pictures were evaluated. Means of the measured distances were calculated and used to estimate the area of PP fat by following the formula for trapezoid areas:

$$
\text { Areasag } p p=\frac{\operatorname{sag} \text { cranial } p p(\mathrm{~cm})+\operatorname{sag} \text { caudal } p p(\mathrm{~cm})}{2} \times 1(\mathrm{~cm})
$$

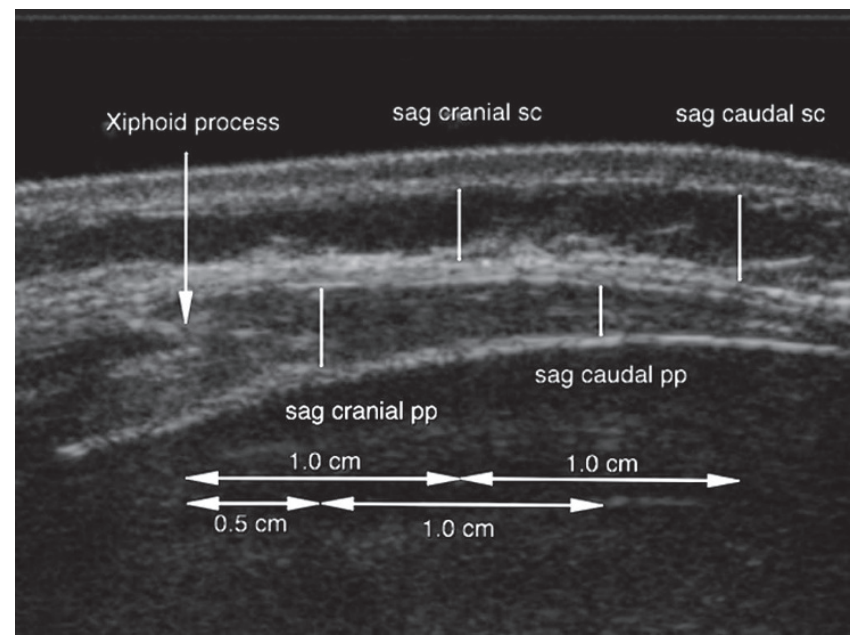

Figure 4. Example for measurements in the sagittal plane with labeling of the xiphoid process (reference structure) and the measurement points sag cranial sc and sag caudal sc (were set 1.0 and $2.0 \mathrm{~cm}$ on the right of the reference structure, respectively) as well as sag cranial pp and sag caudal $\mathrm{pp}$ (were set 0.5 and $1.5 \mathrm{~cm}$ on the right of the reference structure, respectively); areas are calculated by the formula of trapezoid.
Subcutaneous fat. The SC fat layer was determined in sagittal and axial plane. Fat layers were defined as the echo-poor space between the echo-rich cutis and the echo-rich linea alba or the M. rectus abdominis, respectively.

In sagittal plane, the first reference point was set $1.0 \mathrm{~cm}$ caudal the xyphoid process, the lower margin of the sternum (sag cranial sc) and the second reference point $1.0 \mathrm{~cm}$ caudal of the first reference point (sag caudal sc), with highest parallelism of the layers (Figure 4).

In axial plane, the first measurement point was set directly above the linea alba $(\mathrm{ax} \mathrm{m})$ as well as $1.0 \mathrm{~cm}$ on the right $(\mathrm{ax} \mathrm{r})$ and left (ax l) of the linea alba between the cutis and the M. rectus abdominis (Figure 5).

In sagittal and axial plane, three pictures were evaluated. Means of the measured distances were calculated and used to estimate the area of SC fat by using the formula:

$$
\text { Areasag } s c=\frac{\operatorname{sagcranial} s c(\mathrm{~cm})+\operatorname{sag} \operatorname{caudal} s c(\mathrm{~cm})}{2} \times 1(\mathrm{~cm})
$$

\section{Areaaxsc $=$}

$$
\left(\frac{\operatorname{axr}(\mathrm{cm})+\operatorname{axm}(\mathrm{cm})}{2} \times 1(\mathrm{~cm})+\frac{\operatorname{axl}(\mathrm{cm})+\operatorname{axm}(\mathrm{cm})}{2} \times 1(\mathrm{~cm})\right) / 2
$$

Moreover, the ratio of PP and SC fat tissue from the sagittal plane was calculated:

$$
\text { Ratio } P P / S C=\frac{\text { Areasag } P p}{\text { Areasag } s C}
$$

The technique used was a method originally described by Holzhauer and colleagues (12) with small modifications to assess abdominal fat distribution in children under the age of one. Holzhauer et al. (12) suggest calculating the area with the length of $2.0 \mathrm{~cm}$ in sagittal plane. However, this evaluation was not deemed appropriate for the age group $<1 \mathrm{y}$ of age. Therefore, we chose a length of $1.0 \mathrm{~cm}$ for the calculation of the fat areas. In addition, SC fat layer was determined in sagittal and axial plane, respectively.

\section{Reproducibility}

To calculate intra-observer agreement, the investigations of 12 infants ( $n=4$ from each time point), were used and analyzed with OsiriX software by one examiner (E.H.) twice.

For the assessment of inter-observer variation, 45 randomly chosen ultrasound measurements were independently evaluated with the software by two observers (E.H. and D.M.).

For intra- and inter-observer variation, the examiners independently evaluated three pictures and the fat areas were calculated.

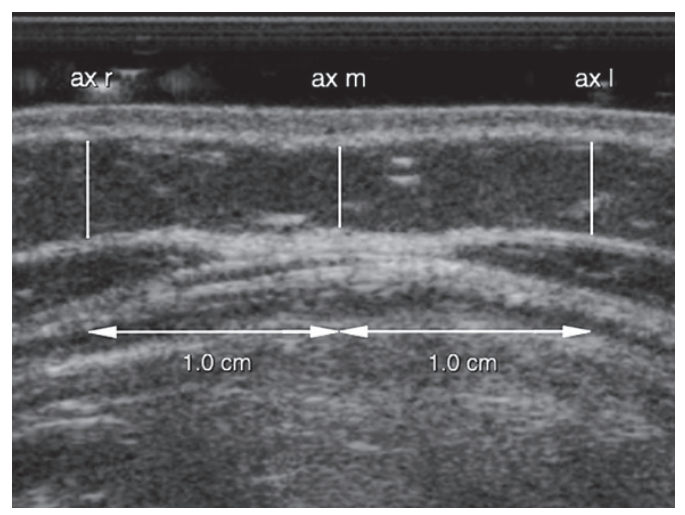

Figure 5. Example for measurements in the axial plane. The measurement is performed directly above the linea alba (ax $\mathrm{m}$ ) as well as $1.0 \mathrm{~cm}$ on the right ( $a x r)$ and 1.0 left ( $a x I)$ midway between the xiphoid process and the umbilicus; areas are calculated by the formula of trapezoid. 


\section{Statistical Analysis}

Summary ultrasound data, anthropometric data and SFT are presented for infants at $6 \mathrm{wk}, 4 \mathrm{mo}$, and $1 \mathrm{y}$. Mixed linear models (using an unstructured covariance matrix) were fitted to these repeated measures with time as a fixed effect. To explore how changes over time differ according to sex, sex was added as a fixed effect in the model together with an interaction between sex and time. Estimated mean differences in sex are presented for each measure at each time point, together with $95 \%$ confidence intervals. Associations between anthropometric and ultrasound variables were assessed using Spearman-Rho correlation coefficient.

Intra- and inter-observer agreements were examined using ICC and their 95\% confidence intervals. An ICC of 1 indicates that all of the observed variation is caused by between subject variations. Additionally, we performed Bland-Altman plots of the areas sag pp, sag sc and ax sc. Statistical analyses were performed using PASW software (version 21, SPSS, Chicago, IL). A two-sided $P$ value $<0.05$ was considered statistically significant, and no correction was made for multiple comparisons.

\section{ACKNOWLEDGMENTS}

We would like to thank E. Rummeny, head of the Institute of Radiology, for giving us free access to the sonographic system.

\section{STATEMENT OF FINANCIAL SUPPORT}

C.B., D.M., E.H., V.S., S.B., L.S., C.V., J.S.B., U.A.G., and H.H. declare financial support to their institutions for the submitted work from the Else Kröner-Fresenius Foundation, Bad Homburg; further financial support by the International Unilever Foundation, Hamburg, by the EU-funded EARNEST (Early Nutrition Programming Project) consortium (FOOD-CT-2005-007036), administratively facilitated by Frank Wiens, and the German Ministry of Education and Research via the Competence Network Obesity (Kompetenznetz Adipositas, 01Gl0842). There was no intervention from any sponsor with any of the research aspects of the study including study design, intervention, data collection, data analysis and interpretation as well as writing of the manuscript.

Disclosures: Danone Research - Centre for Specialised Nutrition, without any involvement in data analysis. The authors have no conflict of interest relevant to this article to disclose.

\section{REFERENCES}

1. Ebbeling CB, Pawlak DB, Ludwig DS. Childhood obesity: public-health crisis, common sense cure. Lancet 2002;360:473-82.

2. Péneau S, Rouchaud A, Rolland-Cachera MF, Arnault N, Hercberg S, Castetbon K. Body size and growth from birth to 2 years and risk of overweight at 7-9 years. Int J Pediatr Obes 2011;6:e162-9.

3. Kissebah AH, Krakower GR. Regional adiposity and morbidity. Physiol Rev 1994;74:761-811.

4. Wajchenberg BL. Subcutaneous and visceral adipose tissue: their relation to the metabolic syndrome. Endocr Rev 2000;21:697-738.

5. Samara A, Ventura EE, Alfadda AA, Goran MI. Use of MRI and CT for fat imaging in children and youth: what have we learned about obesity, fat distribution and metabolic disease risk? Obes Rev 2012;13:723-32.

6. Dencker M, Wollmer P, Karlsson MK, Lindén C, Andersen LB, Thorsson O. Body fat, abdominal fat and body fat distribution related to cardiovascular risk factors in prepubertal children. Acta Paediatr 2012;101:852-7.

7. He Q, Zhang X, He S, et al. Higher insulin, triglycerides, and blood pressure with greater trunk fat in Tanner 1 Chinese. Obesity (Silver Spring) 2007;15:1004-11.

8. Brambilla P, Bedogni G, Moreno LA, et al. Crossvalidation of anthropometry against magnetic resonance imaging for the assessment of visceral and subcutaneous adipose tissue in children. Int J Obes (Lond) 2006;30:23-30.

9. Benfield LL, Fox KR, Peters DM, et al. Magnetic resonance imaging of abdominal adiposity in a large cohort of British children. Int J Obes (Lond) 2008;32:91-9.

10. Wells JC, Fewtrell MS. Measuring body composition. Arch Dis Child 2006;91:612-7.

11. Semiz S, Ozgören E, Sabir N. Comparison of ultrasonographic and anthropometric methods to assess body fat in childhood obesity. Int J Obes (Lond) 2007;31:53-8.
12. Holzhauer S, Zwijsen RM, Jaddoe VW, et al. Sonographic assessment of abdominal fat distribution in infancy. Eur J Epidemiol 2009;24:521-9.

13. Suzuki R, Watanabe S, Hirai Y, et al. Abdominal wall fat index, estimated by ultrasonography, for assessment of the ratio of visceral fat to subcutaneous fat in the abdomen. Am J Med 1993;95:309-14.

14. Mook-Kanamori DO, Holzhauer S, Hollestein LM, et al. Abdominal fat in children measured by ultrasound and computed tomography. Ultrasound Med Biol 2009;35:1938-46.

15. De Lucia Rolfe E, Modi N, Uthaya S, et al. Ultrasound estimates of visceral and subcutaneous-abdominal adipose tissues in infancy. J Obes 2013;2013:951954.

16. Olhager E, Thuomas KA, Wigström L, Forsum E. Description and evaluation of a method based on magnetic resonance imaging to estimate adipose tissue volume and total body fat in infants. Pediatr Res 1998;44:572-7.

17. Butte NF, Hopkinson JM, Wong WW, Smith EO, Ellis KJ. Body composition during the first 2 years of life: an updated reference. Pediatr Res 2000;47:578-85.

18. Fomon SJ, Haschke F, Ziegler EE, Nelson SE. Body composition of reference children from birth to age 10 years. Am J Clin Nutr 1982;35(5 Suppl):1169-75.

19. Olhager E, Flinke E, Hannerstad U, Forsum E. Studies on human body composition during the first 4 months of life using magnetic resonance imaging and isotope dilution. Pediatr Res 2003;54:906-12.

20. Geer EB, Shen W. Gender differences in insulin resistance, body composition, and energy balance. Gend Med 2009;6 Suppl 1:60-75.

21. Gallagher D, Heymsfield SB, Heo M, Jebb SA, Murgatroyd PR, Sakamoto Y. Healthy percentage body fat ranges: an approach for developing guidelines based on body mass index. Am J Clin Nutr 2000;72:694-701.

22. Huang TT, Johnson MS, Figueroa-Colon R, Dwyer JH, Goran MI. Growth of visceral fat, subcutaneous abdominal fat, and total body fat in children. Obes Res 2001;9:283-9.

23. Arfai K, Pitukcheewanont PD, Goran MI, Tavare CJ, Heller L, Gilsanz V. Bone, muscle, and fat: sex-related differences in prepubertal children. Radiology 2002;224:338-44.

24. Webster-Gandy J, Warren J, Henry CJ. Sexual dimorphism in fat patterning in a sample of 5 to 7 -year-old children in Oxford. Int J Food Sci Nutr 2003;54:467-71.

25. Satake E, Nakagawa Y, Kubota A, Saegusa H, Sano S, Ohzeki T. Age and sex differences in fat distribution in non-obese Japanese children. J Pediatr Endocrinol Metab 2010;23:873-8.

26. Karlsson AK, Kullberg J, Stokland E, et al. Measurements of total and regional body composition in preschool children: A comparison of MRI, DXA, and anthropometric data. Obesity (Silver Spring) 2013;21: 1018-24.

27. Liem ET, De Lucia Rolfe E, L'Abée C, Sauer PJ, Ong KK, Stolk RP. Measuring abdominal adiposity in 6 to 7 -year-old children. Eur J Clin Nutr 2009;63:835-41.

28. Hauner H, Much D, Vollhardt C, et al. Effect of reducing the n-6:n-3 longchain PUFA ratio during pregnancy and lactation on infant adipose tissue growth within the first year of life: an open-label randomized controlled trial. Am J Clin Nutr 2012;95:383-94.

29. Hauner H, Vollhardt C, Schneider KT, Zimmermann A, Schuster T, Amann-Gassner $\mathrm{U}$. The impact of nutritional fatty acids during pregnancy and lactation on early human adipose tissue development. Rationale and design of the INFAT study. Ann Nutr Metab 2009;54:97-103.

30. Weststrate JA, Deurenberg P, van Tinteren H. Indices of body fat distribution and adiposity in Dutch children from birth to 18 years of age. Int J Obes 1989;13:465-77.

31. Haffner SM, Stern MP, Hazuda HP, Pugh J, Patterson JK. Do upper-body and centralized adiposity measure different aspects of regional body-fat distribution? Relationship to non-insulin-dependent diabetes mellitus, lipids, and lipoproteins. Diabetes 1987;36:43-51.

32. Weststrate JA, Deurenberg P. Body composition in children: proposal for a method for calculating body fat percentage from total body density or skinfold-thickness measurements. Am J Clin Nutr 1989;50: 1104-15.

33. De Lucia Rolfe E, Sleigh A, Finucane FM, et al. Ultrasound measurements of visceral and subcutaneous abdominal thickness to predict abdominal adiposity among older men and women. Obesity (Silver Spring) 2010;18:625-31. 